\title{
Pulmonary valvular endocarditis in the patient with pulmonary stenosis: A case report with Real-time 3DTEE\& MDCT-640
}

\author{
Nguyen Tuan $\mathrm{Vu}^{*}$ \\ Pham Ngoc Thach University of Medicine, MEDIC HCMC, Vietnam
}

\begin{abstract}
Pulmonary valvular endocarditis is rare disease, the majority of reported cases previously showed isolated PV endocarditis without clear underlying predisposition factors. We report here a 52 ages female patient with endocarditis of pulmonary valve associated with subvalvular pulmonary aneursm, the underlying pulmonary valvular stenosis and RVOT stenosis were confirmed. The 2DTTE, 3D TEE and MDCT-640 were used to make exactly the diagnosis. Patient was undergoing an uncomplicated surgical procedure.
\end{abstract}

\section{Introduction}

Pulmonary valvular stenosis is usually an isolated congenital anomaly and occurs in $7 \%$ to $12 \%$ of patients with congenital heart diseases $[1,2]$ and accounts for $80-90 \%$ of native ouflow tract obstruction. Severe PS often associated with some degree of subvalvular stenosis resulting from RV hypertrophy. 3DTEE, MRI, Cardiac computed tomography can be used to better define the level of obstruction [3-7].

Bacterial endocarditis is a rare complication of the disease. Pulmonarystenosis with moderate to severe degree is stratified to intermediate to high risk of endocarditis [8].

\section{Case report}

A female patient of 52 year old presented at my hospital by persistent fever for one year, she had had this fever twice daily. Urine infection had been diagnosed and treated by Antibiotics in some hospitals but the fever had been not interrupted. Finally she came to MEDIC HCMC.

Physical examination detected a 3/6 systolic murmur at the 3rd LICS like VSD. In her past history, no pathological finding has been noted.

She was evaluated immediately by a chest $\mathrm{X}$ ray that demonstrated a slight prominence of the left second cardiac arch (Figures 1-4).

The transthoracic echocardiography revealed a hypertropic RV with normal TAPSE $=20 \mathrm{~mm}$, normal LV size and function, $\mathrm{EF}=71 \%$. Vegetation of $11 \times 5 \mathrm{~mm}$ in size that attached to the pulmonary valve, recorded from parasternal shorst axis view TTE.

The pulmonary cusps were doming and reduced opening degree, a severe pulmonary stenosis with $\max$ pressure Gradient $=69 \mathrm{mmhg}$, mean $=42 \mathrm{mmhg}$ was confirmed by continue wave Doppler (Figures 5 and 6 ).

Then Real-time 3DTEE was performed, and we found a mobile vegetation with greater size about $14 \times 7 \mathrm{~mm}$ associated with dilated pulmonary trunk of $35 \mathrm{~mm}$ in diameter and a subpulmonary valve aneurysm of $25 \mathrm{x} 36 \mathrm{~mm}$ in measuring (Figure 7).
CT angiography ( MDCT-640) with IV contrast medium Ultravist, slice thickness $=0.5 \mathrm{~mm}$ was indicated and detected a subpulmonary valve aneurysm of $27 x 38 \mathrm{~mm}$ and dilated pulmonary trunk: Annulus $=27 \mathrm{~mm}$, Trunk $=40-46 \mathrm{~mm}, R P A=14 \mathrm{~mm}, \mathrm{LPA}=27 \mathrm{~mm}$. Furthermore, reduced RVOT size revealed: $9.5 \mathrm{~mm}-10.2 \mathrm{~mm}$ in diameter (Figures 8-11).

Patient underwent uncomplicated surgical repair: opening RVOT, valvuloplasty and suture the perforated pulmonary cusp (this perforation was not seen prior to operation) with a favorable postoperative progress (Figures 12 and 13).

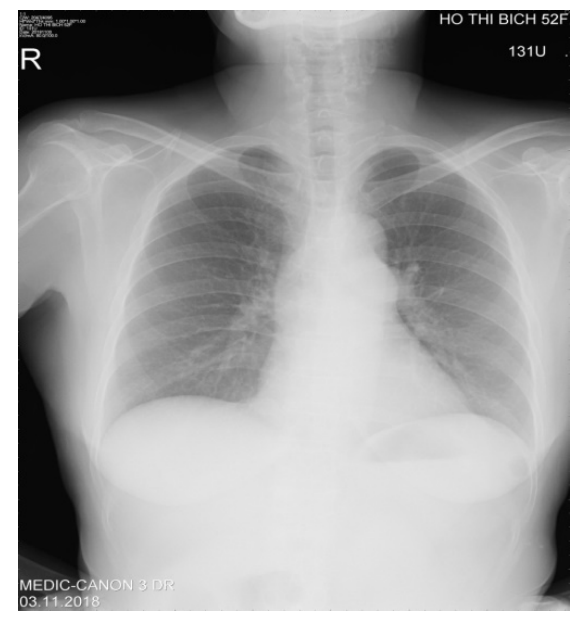

Figure 1. Slightly prominent 2nd left cardiac arch was noted

${ }^{\star}$ Correspondence to: $\mathrm{Vu}$ NT, Pham Ngoc Thach University of Medicine, MEDIC HCMC Vietnam, E-mail: tuanvu2401@yahoo.com

Key words: Pulmonary valvular stenosis, TTE: Transthoracic Echocardiography, TEE: Transesophageal Echocardiography, RT-3DTEE: Real-time-3DTEE, Endocarditis, Vegetation, MDCT: Multidetector Computed Tomography

Received: June 24, 2020; Accepted: June 29, 2020; Published: July 02, 2020 


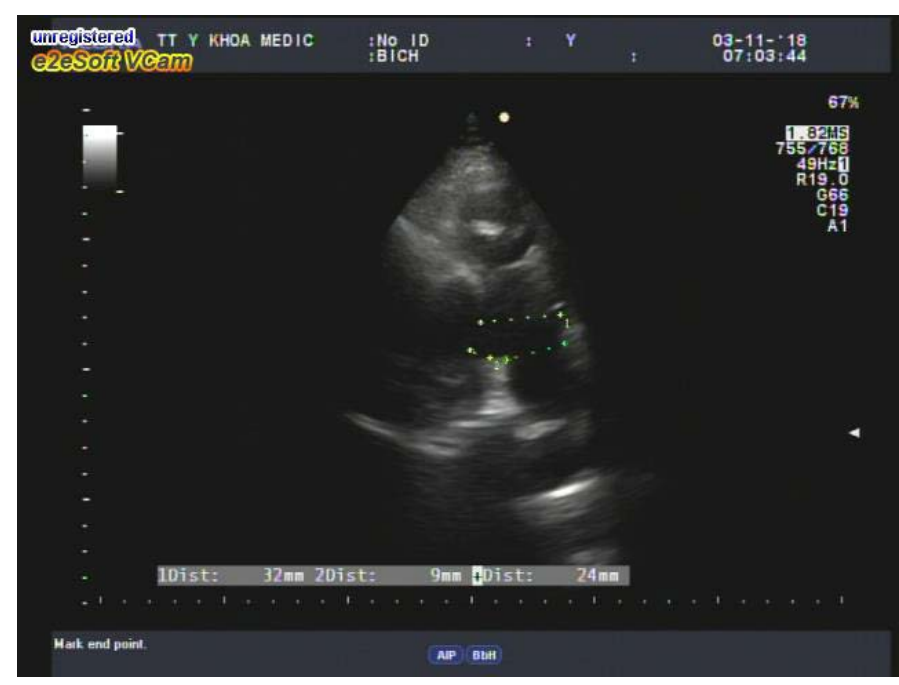

Figure 2. Dilated pulmonary trunk and LPA viewed from 2DTTE parasternal SAX

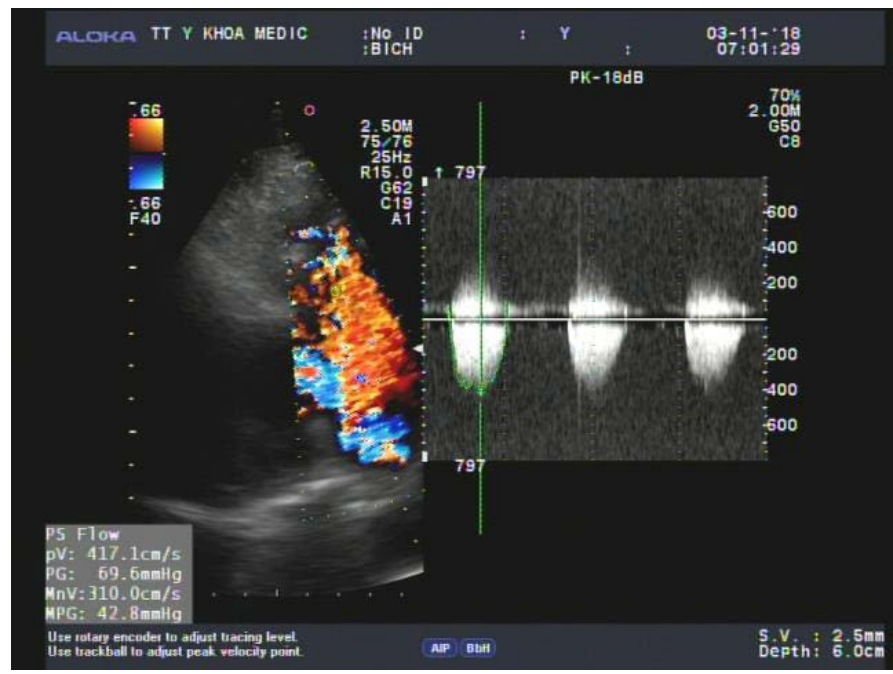

Figure 3. Severe pulmonary stenosis presented by aliasing color Doppler flow and increased transvalvular pressure gradient

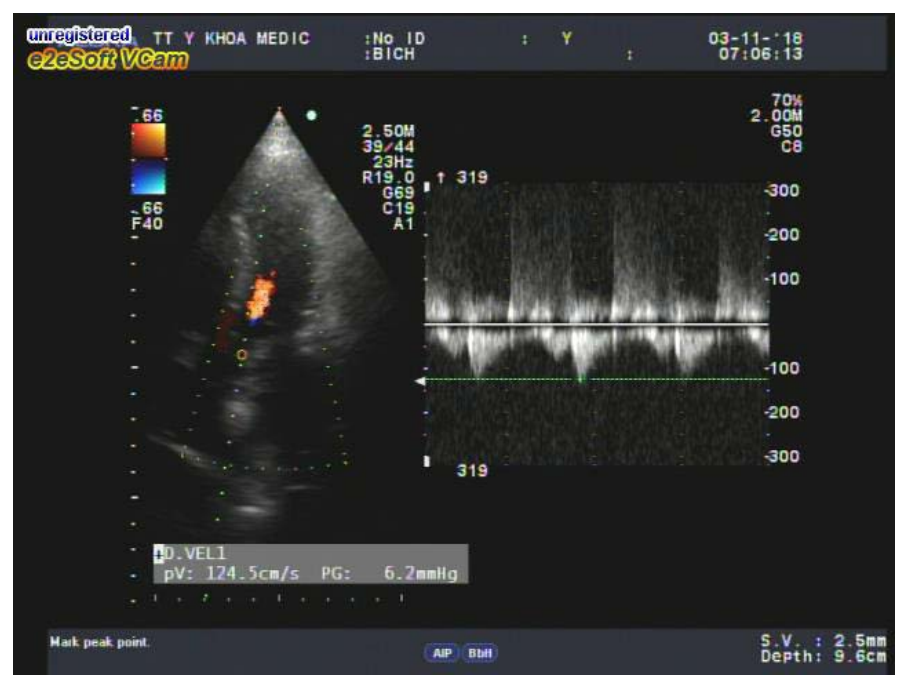

Figure 4. A slight aortic regurgitation recorded from $5 \mathrm{C}$ apical view, no aortic valvular vegetation seen

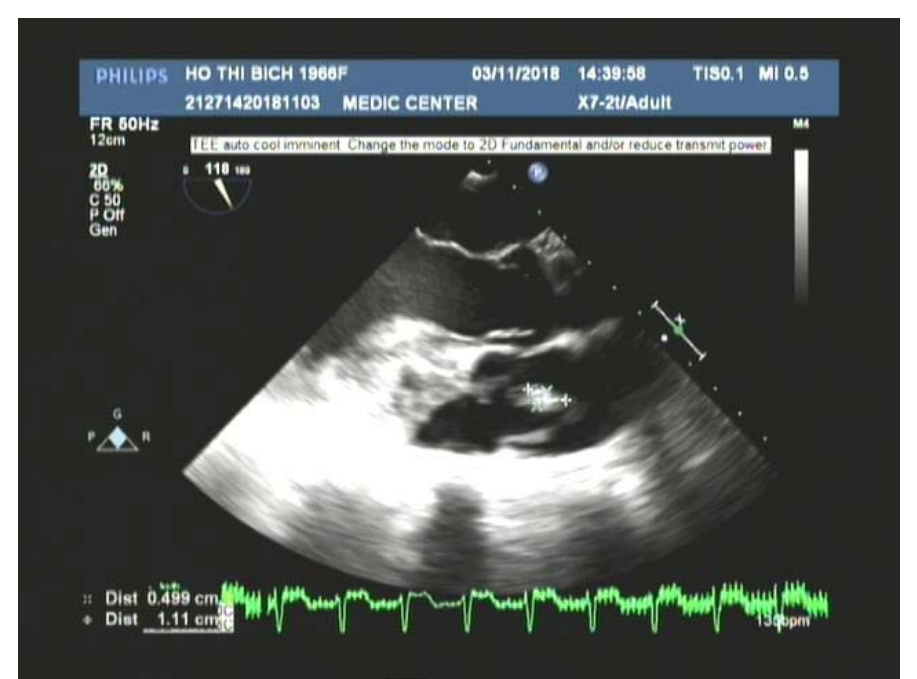

Figure 5. TEE multiplane at $118^{\circ}$ visualized vegetation attached to doming pulmonary valve

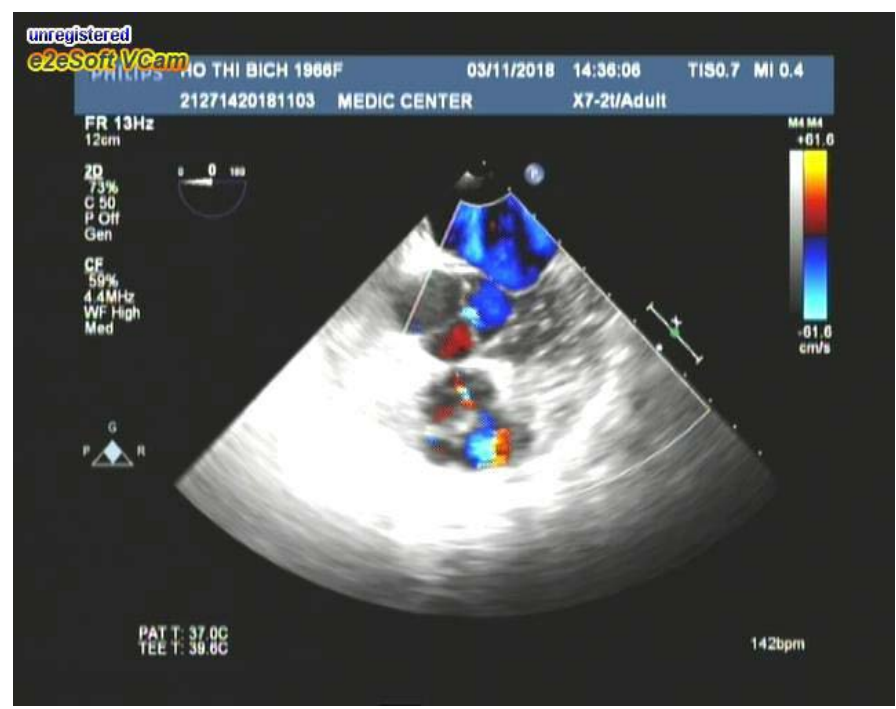

Figure 6. Color Doppler TEE showed PV thickening\& pulmonary regurgitation

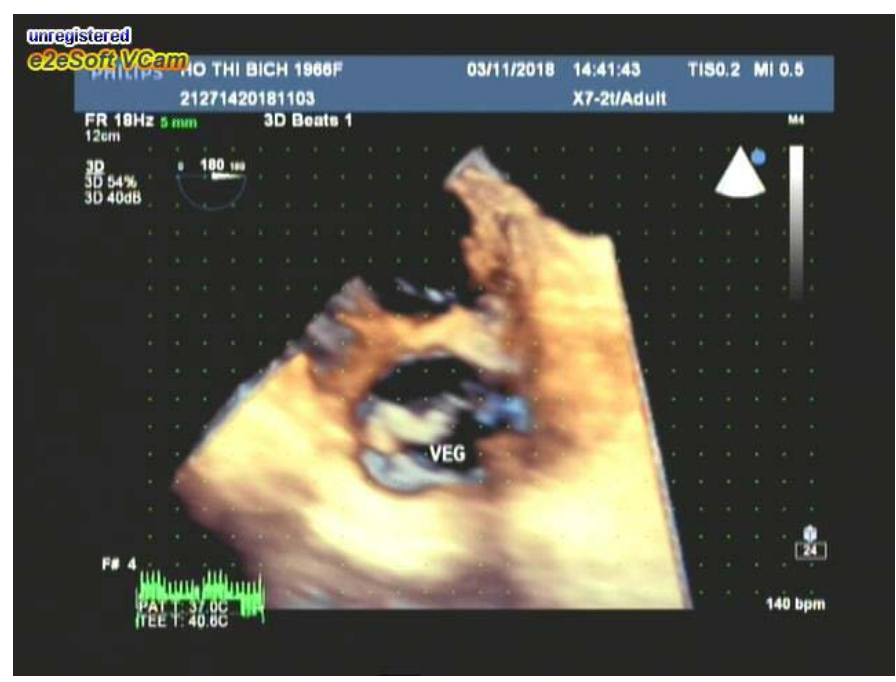

Figure 7. RT-3DTEE demonstrating vegetation better than 2DTEE, particularly shape, size and mobility 


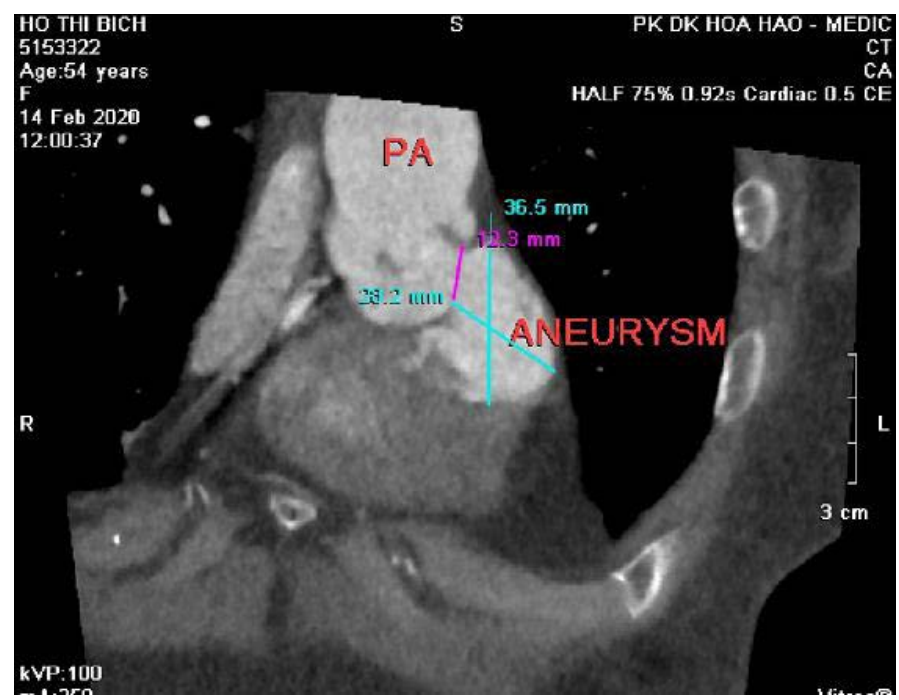

Figure 8. cardiac MDCT with MPR imaging detected a subvalvular pulmonary aneurysm

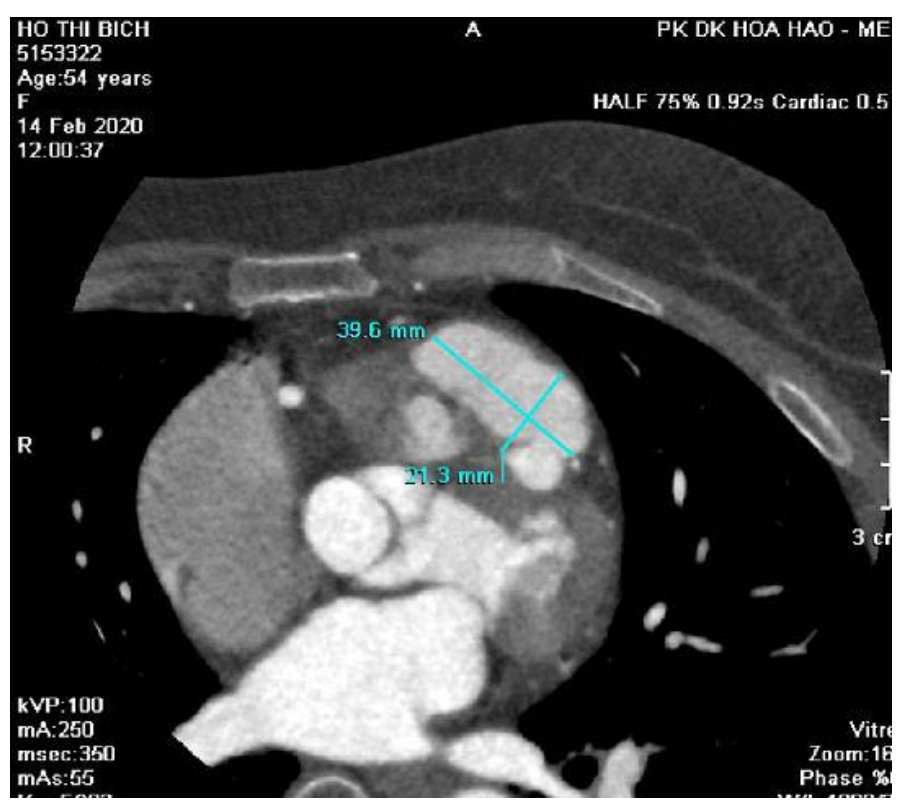

Figure 9. Subvalvular aneursm with contrast medium inside viewed on axial image

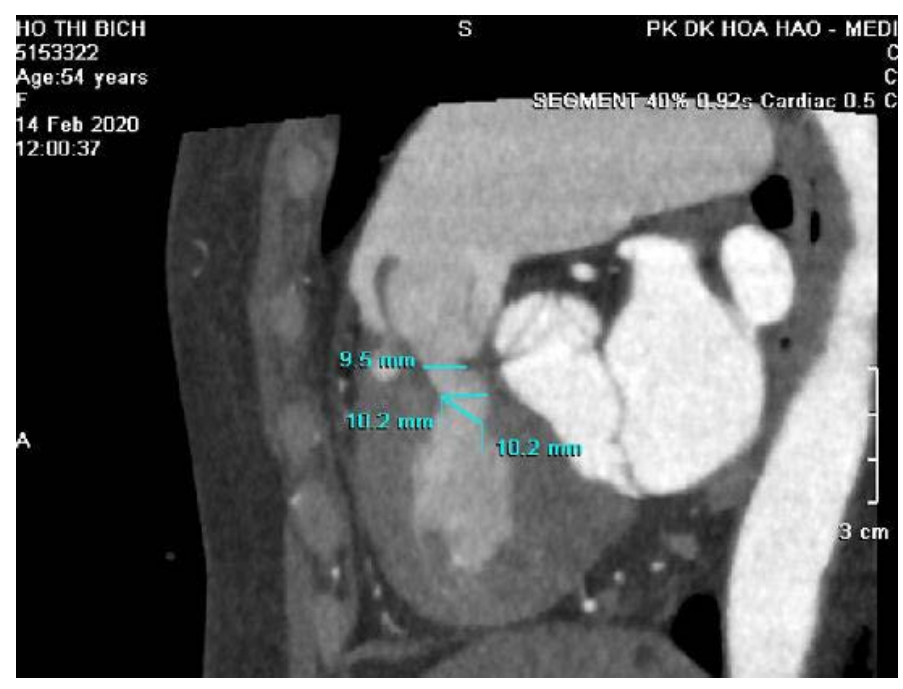

Figure 10. MDCT with sagittal view showed reduced diameter of RVOT

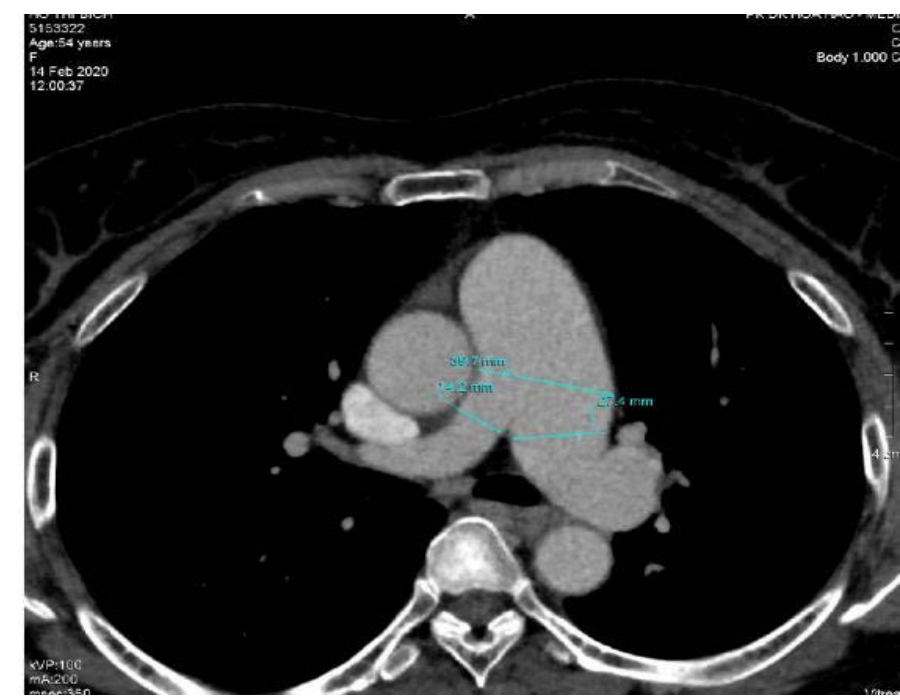

Figure 11. MDCT axial view revealed dilatation of pulmonary trunk and LPA

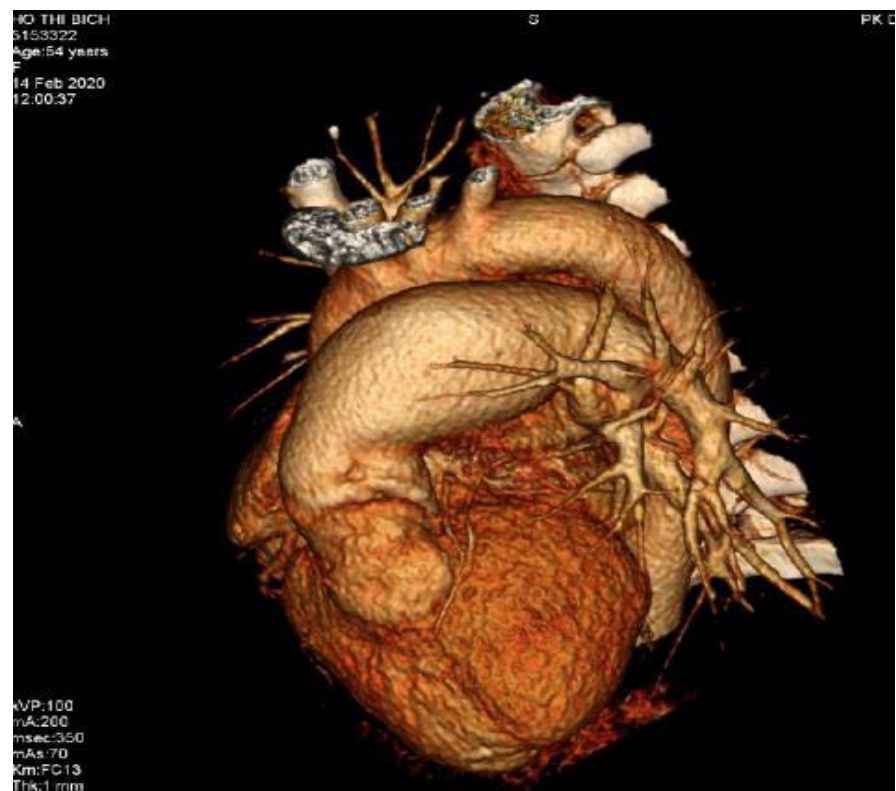

Figure 12. Volume Rendering showed a dilated pulmonary trunk and a subvalvular aneurysm

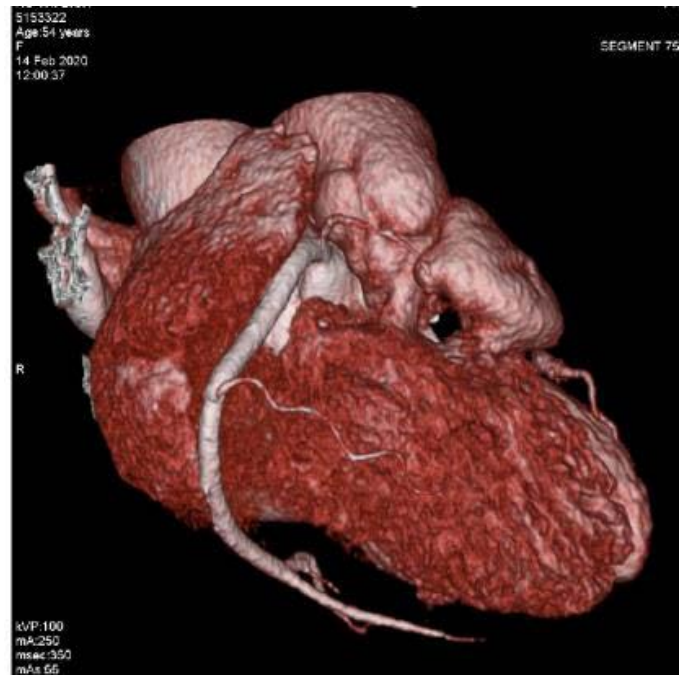

Figure 13. Subvalvular aneurysm viewed from another angle 


\section{Discussion}

Pulmonary valvular infective endocarditis is extremely rare in order to previous publishings. Our patient with pulmonary valvular endocarditis had the underlying prediposition factor including congenital pulmonary valvular stenosis detected on Transthoracic and Transesophageal Echocardiography as domming sign and increased transvalvular pressure gradient.

2DTTE showed a pulmonary valve vegetation that better visualized on the real-time 3DTEE related to its shape, size, mobility. So, 3DTEE provided important informations to make decision of surgical procedure.

MDCT-640 in this case was incated for evaluating 2 level of stenosis: RVOT ( decreasedsize on contrast medium imaging ) and pulmonary valve ( domming sign on systolic imaging ), MDCT also confirme the diagnosis of subpulmonary valve aneurysm that possibly resulted from endocarditis complication.

Siamak M. Seraj, et al. [9] reported an isolated pulmonary valve endocarditis case, his patient 61 year old men, presented in condition of acquired pneumonia. Following positive blood culture, Transthoracic Echocardiography confirmed mobile and large pulmonary valve vegetation and a moderate to severe tricuspid regurgitation.The presence of underlying cause was unclear.

Melissa Lyle, et al. [6] presented a 63 year old man that was hospitalized in setting of arthroplasty of chronic knee pain, the past history of patient included CABG at age 59 . His postoperative course was complicated by an Enterococcus faecalis blood stream infection and aortic valve endocarditis, requiering parenteral antibiotics. Patient then presented again for evaluation of recurrent knee pain, a 2/6 early diastolic murmur at the left upper sternal border was detected. TEE revealed a enlaged vegetation attached to a flail pulmonary cusp associated with severe pulmonary regurgitation. The timing of surgery based on the functional capacity and RV function. The decision was made for continued monitoring.

\section{Conclusion}

Pulmonary valvular endocarditis always are reraly seen and the disease involves to normal as well as abnormal valves like congenital valvular stenosis in this case.

PV endocarditis could be missed if patients do not present typical clinical features of endocarditis, especially when the predisposition risk factors are absent or patients abused antibiotics before presented in hospital.

The diagnosis of endocarditis mainly based on hemoculture and detecting vegetation. Transthoracic Echocardiography completed by Transesophageal Echocardiography, particularly RT-3DTEE better demonstrating the vegetation.

Cardiac MDCT is useful when many levels of obstruction have to been evaluated or endocarditis resulting to severe complications as perforation, aneurysm.

\section{References}

1. Armstrong WF, Ryan T (2010)Feigenbaum's Echocardiography. (7 Edn) William \&Wilkins pp: 361-384

2. Gatzoulis MA, Webb GD, Daubenney PEF (2018) Diagnosis and Management of Adult Congenital Heart Disease. (3 ${ }^{\text {th }}$ Edn) Elsevier, pp: 460-464.

3. Haaga JR, Boll DT (2017) CT and MRI of the whole body. ( $6^{\text {th }}$ Edn) Elservier, p. 2043

4. Hutchison SJ, Merchant N (2015)Principles of cardiac and vascular computed tomography. (1 Edn) Elservier Saunder, pp: 271- 288.

5. Lang RM (2012) EAE,ASE Recommendations for Image Acquisition and Display Using Three-Dimensional Echocardiography. JAm Soc Echocardiography 25: 3-46.

6. Lyle M, Espinosa R (2018) Pulmonary Valve Endocarditis. JACC.

7. Otto C (2017) The Practice of Clinical Echocardiography. ( $5^{\text {th }}$ Edn) Elsevier, pp: 651673.

8. Perloff JK (2009) Congenital Heart Disease in Adults. ( $3^{\text {rd }}$ Edn) Saunsers Elservier, pp: 168-189.

9. Seraj SM, Gill EP, Sekhon S (2017) Isolated pulmonary valve endocarditis: truth or myth. J community hospital internal medicine perspectives $7: 329-331$.

Copyright: (C2020 Nguyen Tuan Vu. This is an open-access article distributed under the terms of the Creative Commons Attribution License, which permits unrestricted use, distribution, and reproduction in any medium, provided the original author and source are credited. 